\title{
Darunavir/cobicistat maintains the effectiveness of darunavir/ritonavir in HIV-infected patients under mono or dual therapy
}

\author{
Álvaro Mena ${ }^{1,2}$, Purificación Cid ${ }^{1,3}$, Carlos Dueñas ${ }^{4}$, María Ángeles Garcinuño ${ }^{5}$, Juan \\ Francisco Lorenzo ${ }^{6}$, Luis Margusino ${ }^{1,3}$, Marina Quiñones ${ }^{6}$, Carmen Grande ${ }^{5}$, Iria \\ Rodríguez-Osorio $^{1,2}$ and Ángeles Castro ${ }^{1,2}$
}

${ }^{1}$ Grupo de Virología Clínica, Instituto de Investigación Biomédica de A Coruña (INIBIC)-Complexo Hospitalario Universitario de A Coruña (CHUAC), Sergas, Universidade da Coruña (UDC), A Coruña, Spain; ${ }^{2}$ Unidad de Enfermedades Infecciosas, Servicio de Medicina Interna, Complexo Hospitalario Universitario de A Coruña (CHUAC), Sergas, A Coruña, Spain; ${ }^{3}$ Servicio de Farmacia, Complexo Hospitalario Universitario de A Coruña (CHUAC), Sergas, A Coruña, Spain; ${ }^{4}$ Unidad de Enfermedades Infecciosas, Servicio Medicina Interna, Hospital Clínico Universitario de Valladolid, Valladolid, Spain; ${ }^{5}$ Servicio de Medicina Interna, Hospital Nuestra Señora de Sonsoles Ávila, Ávila, Spain; ${ }^{6}$ Unidad de Enfermedades Infecciosas, Servicio Medicina Interna, H. Universitario de Burgos, Burgos, Spain

\begin{abstract}
Objectives: Darunavir/ritonavir (DRV/r) in mono or dual therapy has proven efficacy in selected patients. The aim of this study was to evaluate the efficacy of switching from DRV/r to DRV/cobicistat (DRV/c) in patients under mono or dual therapy.

Methods: This was a prospective multicenter cohort study of patients using DRV/r under mono or dual therapy plus lamivudine who changed to DRV/c maintaining the previous regimen. All patients had a controlled HIV viral load ( $<50$ copies $/ \mathrm{ml}$ ) when switched and were examined every 12 weeks. The primary end-point was the percentage of participants without virological failure (VF) at week 48 in the intent-to-treat analysis. The CD4 cell count and concentrations of cholesterol, triglyceride, and creatinine were measured from baseline to week 48.

Results: A total of 162 patients were included: $68.5 \%$ were men, and their mean age was $46 \pm 12$ years. Seventy (43.2\%) patients were treated with DRV/r monotherapy, and $92(56.8 \%)$ were treated with DRV/r plus lamivudine. The efficacy at week 48 was $95.1 \%$ (95\% CI: 90.6\%-97.5\%) in the intent-to-treat analysis and 98.7\% (95.5-99.6\%) in the on-treatment analysis. Two VFs were documented but without development of resistance mutations. No significant changes were found in the lipid profile. Creatinine concentration increased significantly by $0.07 \mathrm{mg} / \mathrm{dl}$ $(0.04-0.10, P<0.001)$.

Conclusions: Switching from DRV/r to DRV/c in patients under mono or dual therapy is safe and effective.
\end{abstract}

Keywords: Darunavir/cobicistat, monotherapy, dual therapy, pharmacokinetics 


\section{Introduction}

The current standard recommendation for HIV treatment is three antiretroviral drugs. The extended use of some NRTIs is associated with adverse events, such as renal and cardiovascular toxicity, and mono and dual therapies are now in use. ${ }^{1,2}$

Boosted PI monotherapy with lopinavir or darunavir (DRV) has proven efficacy in clinical trials and reallife cohort studies when applied in selected patients. ${ }^{3,4}$ In the MONET Study, switching to DRV/ritonavir (DRV/r) monotherapy was as effective in controlling HIV replication as DRV/r plus two NRTIs after 144 weeks (if switches were not considered to be failures). ${ }^{5}$ Dual therapy with DRV/r plus lamivudine (3TC) is a useful switch strategy in HIV-infected patients whose viral replication is controlled over time. $^{6}$

Cobicistat, a cytochrome P450-3A inhibitor, has been developed as a booster for other antiretroviral agents and is used as a co-formulation with DRV, atazanavir and elvitegravir. Cobicistat is a more specific inhibitor than ritonavir, and there are some differences in the drug-interaction profiles. The pharmacokinetics of DRV $800 \mathrm{mg}$ QD administered with $150 \mathrm{mg}$ of cobicistat is bioequivalent to that observed with ritonavir in terms of the AUC and $C_{\max }$, even though the $C_{\min }$ is significantly lower with cobicistat than with ritonavir. ${ }^{7}$

There are few data on the use of DRV/cobicistat (DRV/c) in mono or dual therapies. The aim of this study was to evaluate the efficacy of switching from a DRV/r-based mono or dual therapy (plus 3TC) to a $\mathrm{DRV} / \mathrm{c}$-based while retaining the previous regimen.

\section{Methods}

This was an observational, prospective cohort study of HIV-infected patients treated with a DRV/rcontaining mono or dual therapy (plus 3TC) who switched to DRV/c while maintaining the same regimen. Three clinics in Spain participated in the study; in these clinics, around 2,100 HIV-infected patients are on ART. The research protocol was reviewed and approved by the Regional Ethics Committee (register code 2014/500). All clinical data were anonymized and de-identified prior to be analysis, the identification numbers of the patients were blinded.

All patients included were aged $>18$ years and had an HIV RNA $<50$ copies/ml at the time of the switch. Patients were under mono or dual therapy as usual clinical practice and have to be taking the same treatment at least 3 months before switching to DRV/c.

The epidemiological and clinical data, treatment exposure and reasons for mono or dual therapy were recorded. Patients with detectable HCV-RNA at baseline were considered HCV-coinfected. Patients were followed up every 12 weeks until they either changed or discontinued the treatment, or to the last observation for patients who did not interrupt their treatment. During the follow-up, virological and immunological data were collected. The estimated glomerular filtration rate (eGFR) was calculated using the CKD Epidemiology Collaboration (CKD-EPI) formula. For patients who interrupted the treatment, the date and reason for discontinuation were also registered. Adverse events were categorized via the standardized toxicity grade scale of the 2.1 version of the Division of AIDS (DAIDS). ${ }^{8}$

Virological failure (VF) was defined as two consecutive HIV-RNA measurements $>50$ copies $/ \mathrm{ml}$ or one detectable HIV RNA measurement made at the last visit. A resistance study was performed in patients with VF.

Adherence to ART was measured by self-report and pill count (calculated by dividing the number of doses left to the patient by the number of doses dispensed). 
The primary end-point was the percentage of participants without VF at week 48 analyzed with an intent-to-treat approach (incomplete/missing data = failure). The secondary end-points were the efficacy in an on-treatment analysis. The changes in CD4 cell count and fasting lipid and creatinine concentrations were analyzed.

Continuous variables are reported as mean \pm standard deviation (SD) or median (range), as indicated. For categorical variables, absolute numbers and percentages were computed. Changes in continuous measures from baseline to week 48 were analyzed using the Wilcoxon signed-rank test. The primary endpoint is expressed using the two-sided $95 \% \mathrm{CI}$.

\section{Results}

A total of 162 patients whose medication was switched from mono or dual therapy (plus 3TC) containing DRV/r (all 800/100 mg QD) to one containing DRV/c were included, and followed up to week 48. During the inclusion period, 171 patients changed the therapy, of which 162 patients $(94.7 \%)$ accepted to participate. The main characteristics of the study population are shown in Table 1.

Table 1 Main characteristics of the study population and laboratory parameters during the follow-up

\begin{tabular}{|c|c|c|c|c|c|}
\hline & $\mathrm{Basal}^{\mathrm{a}}$ & Week 24 & Week 48 & $\Delta_{\text {basal-w48 }}{ }^{\mathrm{b}}$ & $P$ value \\
\hline Age (years) & $46 \pm 12$ & & & & \\
\hline \multicolumn{6}{|l|}{ Transmission route } \\
\hline IDU $(\%)$ & $36(22.2)$ & & & & \\
\hline Sexual $(\%)$ & $126(77.8)$ & & & & \\
\hline Length of HIV infection (years) & $12(6-17)$ & & & & \\
\hline HCV coinfection $(\%)$ & $32(20.4)$ & & & & \\
\hline Time on ART (years) & $9.9 \pm 4.0$ & & & & \\
\hline Time on LDR (years) & $2.3 \pm 1.1$ & & & & \\
\hline \multicolumn{6}{|l|}{ Treatment } \\
\hline DRV/c monotherapy (\%) & $70(43.2)$ & & & & \\
\hline LDL-cholesterol (mg/dl) & $128 \pm 22$ & $124 \pm 20$ & $125 \pm 28$ & $-2(-7,+4)$ & 0.416 \\
\hline HDL-cholesterol (mg/dl) & $44 \pm 14$ & $43 \pm 13$ & $42 \pm 12$ & $-3(-5,+1)$ & 0.073 \\
\hline Triglycerides (mg/dl) & $172 \pm 96$ & $165 \pm 75$ & $162 \pm 90$ & $-5(-26,+8)$ & 0.096 \\
\hline Creatinine $(\mathrm{mg} / \mathrm{dl})$ & $1.03 \pm 0.10$ & $1.09 \pm 0.10$ & $1.08 \pm 0.14$ & $+0.07(0.04,0.10)$ & $<0.001$ \\
\hline $\mathrm{eGFR}\left(\mathrm{ml} / \mathrm{min} / 1.73 \mathrm{~m}^{2}\right)$ & $87.4 \pm 10.2$ & $83.1 \pm 9.7$ & $83.8 \pm 11.0$ & $-4.0(-6.3,-0.9)$ & $<0.001$ \\
\hline
\end{tabular}

IDU, intravenous drug use; ART, antiretroviral treatment; LDR, less drugs regimen; DRV/c, darunavir/cobicistat; 3TC, lamivudine; eGFR, estimated glomerular filtration rate (CKD-EPI).

${ }^{\text {a }}$ Last determination before switching.

${ }^{\mathrm{b}}$ Change from basal to week 48 , expressed with the $95 \% \mathrm{CI}$.

The reasons for mono or dual therapy use were: NRTI toxicity (44.4\%), simplification strategy $(33.9 \%)$, patient's wishes $(11.7 \%)$, and other reasons $(10.0 \%)$.

The efficacy values at week 48 were $95.1 \%$ (95\% CI: 90.6-97.5\%) in the intent-to-treat analysis and $98.7 \%(95.5 \%-99.6 \%)$ in the on-treatment analysis. Figure 1 shows the percentages of patients without VF in weeks 24 and 48. 


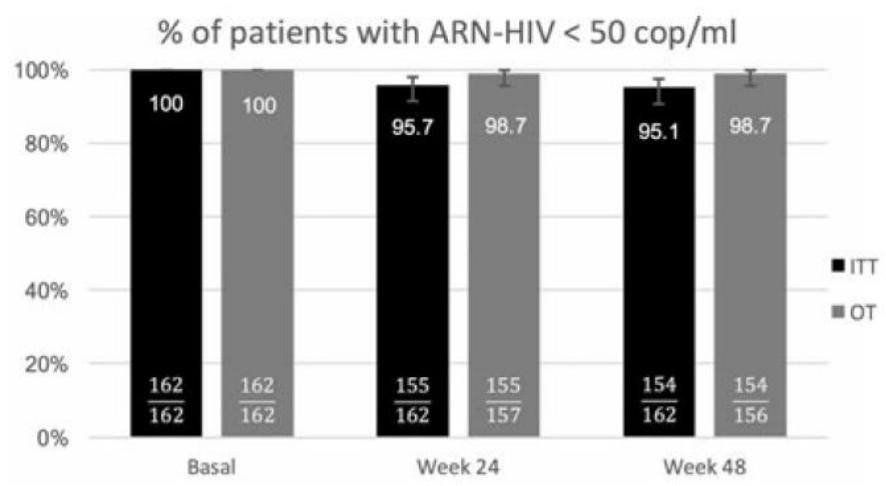

Figure 1 Percentage of patients with HIV viral load $<50$ copies/ml during treatment with DRV/r (basal) and at various times after switching to DRV/c. The intent-to-treat (ITT) and on-treatment (OT) analysis are presented; number inside the bars indicate the \% of patients without viral failure (top numbers) and the number of patients without viral failure (numerators) of the total analyzed (denominators); error bars indicate $95 \%$ CIs.

Eight patients discontinued the DRV/c-based mono or dual therapy. Two were considered to have VF and the other six discontinued for other reasons: three patients returned to DRV/r in the same mono or dual therapy because of gastrointestinal disturbances before week 12; one patient developed peripheral neuropathy (although the investigator did not associate this with DRV/c, the treatment was changed); one patient developed non-Hodgkin lymphoma and switched to another regimen to avoid drug interactions; and one patients was lost to follow-up in week 36 visit. No other clinical or laboratory severe adverse events (grade 3-4) were reported. Serum creatinine concentration increased significantly from baseline to week 12 after switching and stabilized later, and the lipid profile did not change significantly (Table 1). Eighteen patients had basal eGFR $<60 \mathrm{ml} / \mathrm{min} / 1.73 \mathrm{~m}_{2}$ (mean $50 \pm 6 \mathrm{ml} / \mathrm{min} / 1.73 \mathrm{~m}^{2}$ ); the increase in serum creatinine in patients with moderate renal dysfunction was similar to that observed in those with a rate $>60 \mathrm{ml} / \mathrm{min} / 1.73 \mathrm{~m}^{2}(6.0 \%$ vs. $5.7 \%$, respectively).

In one of the two patients with VF, the HIV-RNA was 10,600 copies/ml in week 24; this patient acknowledged treatment withdrawal, without new resistance to PI in the test, and treatment with DRV/c plus two NRTIs was restarted. The other patient was on DRV/c monotherapy and had an HIV-RNA load of 320 copies $/ \mathrm{ml}$ in week 12 , which remained detectable 10 days later (198 copies $/ \mathrm{ml}$ ). Subsequently two NRTIs were introduced, and the next viral load was undetectable, but it was not possible to perform a resistance study; this patient's adherence during the monotherapy regimen was $70 \%-80 \%$.

\section{Discussion}

Our findings suggest that switching from DRV/r to DRV/c in a mono or dual therapy plus 3TC was safe and effective after up to 48 weeks after change. To our knowledge, this is the first study to evaluate this switching strategy in mono and dual therapy receiving patients. The high efficacy obtained is in concordance with data of other study with patients under monotherapy carried in Spain, where only the $5.12 \%$ of patients had any viral load $>200$ copies $/ \mathrm{ml}$ after a median follow-up of 7.93 months. ${ }^{9}$ Despite it, due to the higher rates of virologic failure, PI monotherapy is not recommended as a simplification strategy nowadays.

We found no significant changes in the lipid profile during the follow-up. Previous studies have reported similar lipid profiles for patients treated with DRV/r versus DRV/c, although recent data show an improvement in triglycerides of patients with basal hypertriglyceridemia that change from ritonavir to cobicistat. ${ }^{10}$ Cobicistat, in vitro, promotes less insulin resistance and lipid accumulation than ritonavir, offering a benefit even not clinically evident. ${ }^{11,12}$ 
Creatinine concentration increased slightly in our patients $(0.07 \mathrm{mg} / \mathrm{dl})$ during the first weeks after switching and plateaued during the following weeks, with a median descent of eGFR of $4.0 \mathrm{ml} / \mathrm{min} / 1.73$ $\mathrm{m}^{2}$, pattern that is consistent with the inhibitory effects of cobicistat on renal tubular creatinine secretion, which is not an indicator of renal dysfunction. ${ }^{11}$ Other studies have reported increases in creatinine concentration when cobicistat is used with DRV, atazanavir or elvitegravir, and have reported that this increase is of a similar magnitude for all drugs. ${ }^{13-15} \mathrm{We}$ found similar tolerability, effectiveness and effects on renal function in patients with moderate renal dysfunction compared with those having a normal eGFR.

In the pharmacokinetic study in healthy volunteers, published by Kakuda et al, the AUC for DRV 800 $\mathrm{mg}$ once daily boosted with cobicistat was bioequivalent to that with ritonavir, but the mean DRV $C_{\min }$ $(1,330 \mathrm{ng} / \mathrm{ml})$ and mean pre-dose plasma concentration $\left(C_{0}=2,400 \mathrm{ng} / \mathrm{ml}\right)$ are $26 \%-35 \%$ lower when boosted with cobicistat. ${ }^{7}$ When DRV/c is administered with a standard meal, the DRV exposure is increased by $30 \% .^{13}$ The ARTEMIS trial evaluated DRV/r plus two NRTIs in treatment-naïve patients. In the DRV pharmacokinetic study of the ARTEMIS, in week 4, the mean AUC $(69,359 \mathrm{ng} \mathrm{h} / \mathrm{ml}), C_{\min }$ $(1,189 \mathrm{ng} / \mathrm{ml})$, and $C_{0}(1,826 \mathrm{ng} / \mathrm{ml})$ were lower than obtained when DRV is boosted with cobicistat, but the effectiveness was not compromised.7,15 High intra- and interpatient variability have been reported for DRV level. ${ }^{16,17}$ There is no evidence of a relationship between the DRV $C_{\min }$ and $C_{0}$ and the virological response, probably because these concentrations are much higher than the half-maximum effective concentration of DRV for the wild-type virus $\left(\mathrm{EC}_{50}=55 \mathrm{ng} / \mathrm{ml}\right)$ and for resistant $\mathrm{HIV}\left(\mathrm{EC}_{50}=550\right.$ $\mathrm{ng} / \mathrm{ml}){ }^{18}$

Adherence is crucial in patients treated with a mono or dual therapy. Our study had only two patients with VF, one instance of which was secondary to voluntary treatment discontinuation, and this patient was probably not a good candidate for mono or dual therapy. The other VF involved low-level viremia in a patient with adherence $<90 \%$. When treating patients with a DRV/c-containing mono or dual therapy, adherence, interactions and administration with meals must be considered.

Our study has some limitations. First, it was a non-comparative design, even though a randomized clinical trial would have been the ideal option, it was not possible to carry it out; however, this lack of a $\mathrm{DRV} / \mathrm{r}$ arm can penalize the switching strategy because all patients started with an undetectable viral load, besides that the real-life data are also very relevant. Second, the sample size did not allow us to detect uncommon adverse events, but previous studies have determined the safety profile of DRV/c. ${ }^{11}$ Third, we did not include pharmacokinetic data, which would have provided more complete clinical information.

In conclusion, switching from DRV/r to DRV/c in patients under mono or dual therapy was shown to be safe and effective. These data support this switching strategy in most of patients taking a DRV/r containing regimen, with the exception of those who do not tolerate cobicistat and those who have some problem of drug interactions, as well as in pregnancy, where neither the cobicistat nor the mono or dual therapies are indicated.

\section{Conflict of interest}

The authors report no declarations of interest.

\section{Funding}

This work was supported in part by grants from Fondo de Investigación Sanitaria (JR17/00028) and Fundación Profesor Novoa Santos, A Coruña. 


\section{Notes on contributors}

Álvaro Mena coordinated the study, performed research, collected, analyzed and interpreted the data, and wrote the manuscript.

Purificación Cid contributed to the design of the study, the analysis and interpretation of the data, and writing of the manuscript.

Carlos Dueñas, María Ángeles Garcinuño, Juan Francisco Lorenzo, Luis Margusino, Marina Quiñones and Carmen Grande contributed to the collection of the data and writing of the manuscript.

Iria Rodríguez-Osorio contributed to the analysis and interpretation of the data.

Ángeles Castro contributed to the design of the study, the analysis and interpretation of the data, writing of the manuscript and was responsible for the overall supervision.

\section{References}

1. Brennan A, Evans D, Maskew M, et al. Relationship between renal dysfunction, nephrotoxicity and death among HIV adults on tenofovir. AIDS. 2011;25:1603-1609.

2. Marcus JL, Neugebauer RS, Leyden WA, et al. Use of abacavir and risk of cardiovascular disease among HIV-infected individuals. J Acquir Immune Defic Syndr. 2016;71:413-419.

3. Arribas JR, Girard PM, Paton N, et al. Efficacy of protease inhibitor monotherapy vs. triple therapy: meta-analysis of data from 2303 patients in 13 randomized trials. HIV Med. 2016;17:358-367.

4. López-Cortés LF, Castaño MA, López-Ruz MA, et al. Effectiveness of ritonavir-boosted protease inhibitor monotherapy in clinical practice even with previous virological failures to protease inhibitorbased regimens. PLoS One. 2016;11:e0148924.

5. Arribas JR, Clumeck N, Nelson M, et al. The MONET trial: week 144 analysis of the efficacy of darunavir/ritonavir (DRV/r) monotherapy versus DRV/r plus two nucleoside reverse transcriptase inhibitors, for patients with viral load <50 HIV-1 RNA copies/mL at baseline. HIV Med. 2012;13:398405.

6. Pulido F, Ribera E, Lagarde M, et al. Dual therapy with darunavir and ritonavir plus lamivudine vs triple therapy with darunavir and ritonavir plus tenofovir disoproxil fumarate and emtricitabine or abacavir and lamivudine for maintenance of human immunodeficiency virus type 1 viral suppression: randomized, openlabel, noninferiority DUAL-GESIDA 8014-RIS-EST45 Trial. Clin Infect Dis. 2017;65:2112-2118.

7. Kakuda TN, Opsomer M, Timmers M, et al. Pharmacokinetics of darunavir in fixed-dose combination with cobicistat compared with coadministration of darunavir and ritonavir as single agents in healthy volunteers. J Clin Pharmacol. 2014;54:949-957.

8. U.S. Department of Health and Human Services, National Institutes of Health, National Institute of Allergy and Infectious Diseases, Division of AIDS. Division of AIDS (DAIDS). Table for grading the severity of adult and pediatric adverse events. Corrected Version 2.1. Accessed July 2017. Available from: https://rsc.tech-res.com/docs/default-source/safety/division-ofaids-(daids)-table-for-grading-theseverity-of-adult-and-pediatric-adverse-events-corrected-v-2-1.pdf.

9. Yunquera-Romero L, Asensi-Díez R, Del Rio-Valencia JC, et al. Darunavir/cobicistat monotherapy. Experience in a tertiary hospital. Rev Esp Quimioter. 2016;29:308-317.

10. Echeverría P, Bonjoch A, Puig J, et al. Significant improvement in triglyceride levels after switching from ritonavir to cobicistat in suppressed HIV-1-infected subjects with dyslipidaemia. HIV Med. 2017;18:782-786.

11. Tashima K, Crofoot G, Tomaka FL, et al. Cobicistat-boosted darunavir in HIV-1-infected adults: week 48 results of a Phase IIIb, open-label single-arm trial. AIDS Res Ther. 2014;11:39.

12. Xu L, Liu H, Murray BP, et al. Cobicistat (GS-9350): a potent and selective inhibitor of human CYP3A as a novel pharmacoenhancer. ACS Med Chem Lett. 2010;1:209-213.

13. Kakuda TN, Van De Casteele T, Petrovic R, et al. Bioequivalence of a darunavir/cobicistat fixed-dose combination tablet versus single agents and food effect in healthy volunteers. Antivir Ther. 2014;19:597-606.

14. Gallant JE, Koenig E, Andrade-Villanueva JF, Chetchotisakd P, DeJesus E, Antunes F, et al. Brief report: cobicistat compared with ritonavir as a pharmacoenhancer for atazanavir in combination with emtricitabine/tenofovir disoproxil fumarate: week 144 results. J Acquir Immune Defic Syndr. 2015;69:338-340.

15. Sekar V, Vanden Abeele C, Van Baelen B, et al. Pharmacokinetic-pharmacodynamic analyses of oncedaily darunavir-ritonavir in the ARTEMIS study; Alexandria, VA, USA. Foundation for Retrovirology and Human Health; Abstracts of the Fifteenth Conference on Retroviruses and Opportunistic Infections, Boston, MA, 2008. Abstract 769. 
16. Sekar V, De La Rosa G, Van de Casteele T. Pharmacokinetic and pharmacodynamic analyses of onceand twice-daily darunavir/ritonavir in the ODIN trial. J Int AIDS Soc. 2010;13(Suppl 4):P185.

17. Kakuda T, Brochot A, Tomaka F, et al. Generalized additive model analysis of the relationship between darunavir pharmacokinetics and pharmacodynamics following once-daily darunavir/ritonavir 800/100 mg treatment in the Phase III trials ARTEMIS and ODIN. Abstracts of the Eleventh International Congress on Drug Therapy in HIV Infection, Glasgow, 2012. Abstract P072.

18. Post FA, Tebas P, Clarke A, et al. Switching to tenofovir alafenamide, coformulated with elvitegravir, cobicistat, and emtricitabine, in HIV-infected adults with renal impairment: 96-week results from a single-arm, multicenter, open-label phase 3 study. J Acquir Immune Defic Syndr. 2017;74:180-184. 